Risk factors for mortality in Reoperations for Pediatric and Congenital Heart Surgery in a Developing Country

\title{
AUTHORS
}

Carlos A. Villa-Hincapie MD. Fellow of Cardiovascular Surgery. Fundación Cardioinfantil (FCl) - Instituto de Cardiología. Bogotá, Colombia. Universidad del Rosario

Marisol Carreno-Jaimes MD. Msc. Clinical Epidemiologist. Department of Congenital Heart Disease. Fundación Cardioinfantil (FCl) - Instituto de Cardiología Bogotá, Colombia. Universidad del Rosario

Carlos E. Obando-Lopez MD. Cardiovascular Pediatric Surgeon. Department of Congenital Heart Disease. Fundación Cardioinfantil (FCl) - Instituto de Cardiología. Bogotá, Colombia. Universidad del Rosario

Jaime Camacho-Mackenzie MD. Cardiovascular Surgeon. Department of Cardiovascular Surgery. Fundación Cardioinfantil (FCI) - Instituto de Cardiología. Bogotá, Colombia. Universidad del Rosario

Juan P. Umaña-Mallarino MD. Cardiovascular Surgeon. Department of Cardiovascular Surgery. Fundación Cardioinfantil (FCl) - Instituto de Cardiología Bogotá, Colombia. Universidad del Rosario

Nestor F. Sandoval-Reyes MD. Cardiovascular Pediatric Surgeon. Director, Department of Congenital Heart Disease. Fundación Cardioinfantil (FCl) - Instituto de Cardiología. Bogotá, Colombia. Universidad del Rosario 


\section{MEETING PRESENTATION}

This paper was presented at the Joint Meeting of the World Society for Pediatric and Congenital Heart Surgery (WSPCHS) in Cartagena - Colombia, from the 26 to the 28th of February 2015. This work earned the first place in the category of oral presentations. The data was updated for this publication.

\section{KEYWORDS}

Congenital Heart Disease; Congenital Heart Surgery; Reoperation; Surgery, Complications

\section{TOTAL WORD COUNT}

2969 words

\section{CORRESPONDING AUTHOR}

Carlos A. Villa-Hincapie MD. Fundación Cardioinfantil (FCl) - Instituto de Cardiología. Bogotá, Colombia. Address: Calle 163A \#13B - 60. Telephone number: (571) 6672727 - Extension number: 3149. E-mail: carlosandresvilla@hotmail.com. 


\section{ABSTRACT}

Background: The survival of patients with congenital heart disease has increased in the recent years, because of enhanced diagnostic capabilities, better surgical techniques and improved perioperative care. Many patients will require reoperations as part of staged procedures, or to treat grafts deterioration and residual or recurrent lesions. Reoperations favor the formation of cardiac adhesions and consequently increase surgery time, however, the impact on morbidity and operative mortality is certain. The objective of the study was to describe the risk factors for mortality in pediatric patients undergoing a reoperation for congenital heart disease. Methods: Historic cohort of patients who underwent reoperations after pediatric cardiac surgery from January 2009 to December 2015. Operations with previous surgical approach different to sternotomy were excluded from the analysis. Results: In seven years, 3.086 surgeries were performed, 481 were reoperations and 238 patients fulfilled the inclusion criteria. Mean number of prior surgeries was $1.4 \pm 0.6$. Median age at the time of reoperation was 6.4 years. The most common surgical procedures were staged palliation for functionally univentricular heart (17.6\%). Median cross clamp time was 66 minutes. Younger age at the moment of resternotomy, longer cross clamp time and a STAT risk category greater than 3 were risk factors for mortality. The number of resternotomies was not associated to mortality. Mortality prior to hospital discharge was $4.6 \%$ and mortality after discharge but prior to 30 days after surgery was $0.54 \%$. Operative Mortality was $5.1 \%$. Conclusions: Resternotomy in pediatric cardiac surgery is a safe procedure in our center.

Abstract word count: 250 words. 


\section{INTRODUCTION}

The survival of patients with congenital heart disease has increased in the last years, because of enhanced diagnostic capabilities, better surgical techniques and improved perioperative care. Many patients will require reoperations as part of staged procedures, to address residual or recurrent defects, or to manage deterioration or outgrowth of grafts. [1]

Repeat sternotomy favors formation of cardiac adhesions, and it has been questioned if this is associated with increased morbidity and operative mortality. None-the less, in the last twenty years this idea has been studied. [2,3,4] One of the first studies on the subject was carried out by Russel J. et al in 1998, with the conclusion that repeat sternotomy carries minimal added risk. [5] In 2008 Morales et al demonstrated that repeat sternotomy represents a negligible risk of injury and therefore that the choice of management strategies should not be affected by the need of a reoperation. [2] Furthermore, Kirshbom PM et al [6] and t Holst KA at Mayo Clinic [7] showed that reentry injuries are not associated with increased operative mortality and that resternotomy is not an independent predictor of early death. More recently, in 2014, an analysis of the Society of Thoracic Surgeons (STS) Congenital Heart Surgery Database reported that "reoperative cardiac surgery for congenital heart disease is common, with one third of index operations in the database occurring subsequent to prior cardiothoracic operation(s) performed on cardiopulmonary bypass. This analysis suggests that a history of previous cardiac surgery does not independently confer a significant incremental risk of operative mortality, but that patients with greater number of previous operations appear to be at higher risk." [1] 
Several strategies for risk reduction in mortality and morbidity during resternotomy are described:[8] PTFE (Polytetrafluoroethylene) membrane, [3] preoperative chest computed tomography. $[9,10,11,12]$ Use of an oscillating saw $[10,13]$ and peripheral cannulation. $[10,11,14]$ The aim of this study was to describe the experience in reoperations of pediatric patients with $\mathrm{CHD}$ in a center from a low-middle income country and to determine the factors associated with operative mortality.

\section{PATIENTS AND METHODS}

The study design was a historical cohort carried out at the Fundación Cardioinfantil Instituto de Cardiología $(\mathrm{FCl}-\mathrm{IC})$. Information was collected from the pediatric cardiac surgery department's database in a prospective manner. This database was audited and approved by The International Quality Improvement Collaborative (IQIC). All reoperations for CHD carried out at our center between January 2009 and December 2015 were included. Patients with an initial surgical approach different to sternotomy or with the indication for the initial operation as non-cardiac disease were excluded from the analysis.

All patients had a preoperative chest x-ray study and echocardiogram, routine CT and MRI were not used to assess the risk of reoperation. The surgical protocol was standard for all: 1) Use of an oscillating saw for the anterior sternum wall (Fig. 1a), 2) use of scissors for the posterior sternum wall (Fig. 1b) to complete sternotomy and 3) lysis of adhesions to identify safe cannulation sites (Fig. 1c).

Variables chosen were: Patients characteristics (age, sex, weight, arterial oxygen saturation - [SaO2], hematocrit, left ventricular ejection fraction - [LVEF], Down 
Syndrome). Procedural characteristics (type of surgery, cardiopulmonary bypass [CPB], time, Cross clamp time [CCT], open chest, STAT Mortality Category, and number of previous surgeries. The endpoint of analysis was post-operative mortality. All variables were defined by the same criteria established by the STS to avoid bias. Patient follow-up was carried out according to the institutional protocol (by telephone, 45 days after hospital discharge).

An initial descriptive analysis was performed. Quantitative variables were evaluated with the Shapiro Wilk test to determine the distribution of the data. Normal variables were analyzed by means and standard deviation, nonparametric variables with median and interquartile range. Categorical variables were analyzed with relative and absolute frequencies; a bivariate analysis was done to identify factors related with mortality; continuous variables were compared with Mann-Whitney and categorical variables with Pearson's chi-squared test. An unconditional univariate logistic regression model was performed without interactions to describe the Odds Ratio of the variables associated with mortality. It was not possible to perform an appropriate multivariable logistic model since an " $n$ " of 11 events is too small. A cutoff point strategy was applied to continuous variables to achieve a dummy variable and include in the model. All tests were performed using STATA 14.0. TX: StataCorp LP.

This study was conducted according to ethic norms and under the precepts of the Colombian Government Ministerio de Salud y Protección Social (Ministry of Health and Social Protection), according the Resolution 8430, 1993. This study was classified as a harm less investigation. The authors assure data confidentiality. 


\section{RESULTS}

During the study period, 3086 patients were taken to surgery for CHD treatment, 481 were reoperations and 239 patients fulfilled de inclusion criteria. Mean number of prior surgeries was $1.4 \pm 0.6$. Figure 2 documents the annual proportion of primary operations and reoperations from 2009 to 2015, inclusive and reveals that the proportion of reoperations increased in the last 4 years of the study.

Table 1 shows the characteristics of the patients included in the study. The median age of the patients who died was lower than the median age of the patients who survived, however, this difference was not statistically significant. The majority of the patients did not present with anemia, severe hypoxemia, or left ventricular dysfunction. More than $30 \%$ of the patients had more than 2 prior sternotomies, but the number of prior sternotomies was not related to mortality in our study. Most of the patients who died had just one reoperation. There were 15 injuries during resternotomy $(6.3 \%)$ requiring emergent establishment of cardiopulmonary bypass and all of them required blood product transfusion. Emergent cardiopulmonary bypass was not considered to be contributory to the outcome of the patients since none of them died within the first 30 days after surgery. Perfusion time and clamp time were different in the two groups of patients, being longer in the group of patients who died with a statistically significant difference, as a reflection of the complexity of the operation. Mortality was related to a higher STAT Mortality Category, almost one third of the operations overall had a STAT Mortality Category of 4 or 5 , and $54 \%$ of the operations associated with mortality had a STAT Mortality 
category 4 or 5 , with a statistically significant difference in comparison to the patients who survived. As a result of the complexity, open chest was more frequently used in the patients who died.

As demonstrated in Table 2, functionally univentricular heart was the most frequent condition to require reoperation (17.6\%), followed by left heart lesion (12.6\%) such as subaortic membrane and aortic valve pathology, and then surgery for pulmonary valve replacement after repair of Tetralogy of Fallot $(10.1 \%)$. Univariate analysis (Table 3) indicated that younger age at the moment of resternotomy, longer cross clamp time, and a STAT Mortality Category greater than 3 were risk factors for mortality. Resternotomy was not associated with any catastrophic operative events that resulted in the immediate death of any patient. Mortality prior to hospital discharge was $4.6 \%$ and mortality after discharge but prior to 30 days after surgery was $0.54 \%$. Therefore, Operative Mortality was $5.1 \%$. When comparing mortality prior to hospital discharge in the same period for primary sternotomy and repeat sternotomy, $4.2 \%(110 / 2611)$ and $4.6 \%(11 / 239)$ respectively, the $p$-value is 0.77 with a confidence interval of $95 \%$. This shows a non statistical difference between mortality prior to hospital discharge among primary and repeat sternotomy.

\section{COMMENT}

Improvements in the survival of patients with $\mathrm{CHD}$ has increased the number of reoperations, [15] so pediatric cardiac centers should be prepared to deal with this new type of patients. The most common indication for resternotomy in this study was staged palliation for functionally univentricular heart, followed by obstructive left heart 
lesions. This finding is explained by increased prevalence of these type of lesion in Colombia, as was reported in the study by Garcia et al. [16]

This study shows similar results to those published by the Mayo Clinic, Texas Children's Hospital, and the STS Congenital Heart Surgery database which have all demonstrated that repeat sternotomy is not an independent risk factor for operative mortality. Furthermore, it is important to highlight that in our cohort, not only resternotomy, but also the number of repeat sternotomies (up to 4) were not risk factors for operative mortality. This latter finding is different from the results published from the Mayo Clinic and the STS Congenital Heart Surgery Database. [1]

Many centers use preoperative CT in a routine manner or in high risk patients, to develop a surgical plan in order to decrease the probability of cardiac injury during repeat sternotomy. $[9,10,11,12]$ However, this type of preoperative evaluation increases the costs of each procedure and in a health system with limited resources as ours, it represents an additional cost difficult to afford. In this study, we found that preoperative CT is not always necessary to achieve good results, using only a preoperative chest $\mathrm{x}$-ray and angiogram to assess the relationship between cardiac structures and the sternum.

The outcomes of this study are the result of a standardized surgical protocol, standardized intensive care, and quality improvement measurements that ensure teamwork and patient safety. However, as this study had a retrospective cohort variable systematic collection was difficult and it was not possible to analyze other variables detected as relevant in the moment of data analysis. Another limitation of our study is its descriptive nature, because conclusions cannot be generalized to all 
of the population. None the less, our results do demonstrate that reoperations for CHD can be carried out safely in our institution.

\section{SUMMARY}

In our study resternotomy was not an independent risk factor for operative mortality in surgery for CHD. Our institutional protocol of evaluation and care for reoperations has shown good outcomes. In our experience repeat sternotomy, is a safe procedure for the treatment of the patient with CHD in all stages of the disease.

CONFLICT OF INTEREST STATEMENT: None declared. 


\section{REFERENCES}

1. Jacobs JP, Mavroudis C, Quintessenza JA, Chai PJ, Pasquali SK, Hill KD et al. Reoperations for pediatric and congenital heart disease: an analysis of the Society of Thoracic Surgeons(STS) congenital heart surgery database. Semin Thorac Cardiovasc Surg Pediatr Card Surg Annu. 2014;17(1):2-8

2. Morales DL, Zafar F, Arrington KA, Gonzalez SM, McKenzie ED, Heinle JS, et al. Repeat sternotomy in congenital heart surgery: no longer a risk factor. Ann Thorac Surg. 2008 Sep;86(3):897-902; discussion 897-902

3. Jacobs JP, lyer RS, Weston JS, Amato JJ, Elliott MJ, de Leval MR et al. Expanded PTFE membrane to prevent cardiac injury during resternotomy for congenital heart disease. Ann Thorac Surg. 1996 Dec;62(6):1778-82

4. Yin $\mathrm{CH}$, Yan J, Li SJ, Li DY, Wang Q, Wang ES. Effect analysis of repeat sternotomy in pediatric cardiac operations. Effect analysis of repeat sternotomy in pediatric cardiac operations. J Cardiothorac Surg. 2015 Nov 30;10:179

5. Russell JL, LeBlanc JG, Sett SS, Potts JE. Risks of repeat sternotomy in pediatric cardiac operations. Ann Thorac Surg. 1998 Nov;66(5):1575-8

6. Kirshbom PM, Myung RJ, Simsic JM, Kramer ZB, Leong T, Kogon BE et al. One thousand repeat sternotomies for congenital cardiac surgery: risk factors for reentry injury. Ann Thorac Surg. 2009 Jul;88(1):158-61 
7. Holst KA, Dearani JA, Burkhart HM, Connolly HM, Warnes CA, Li Z et al. Risk factors and early outcomes of multiple reoperations in adults with congenital heart disease. Ann Thorac Surg. 2011 Jul;92(1):122-8; discussion 129-30

8. Imran Hamid U, Digney R, Soo L, Leung S, Graham AN. Incidence and outcome of re-entry injury in redo cardiac surgery: benefits of preoperative planning. Eur $\mathrm{J}$ Cardiothorac Surg. 2015 May;47(5):819-23

9. Lapar DJ, Ailawadi G, Irvine JN Jr, Lau CL, Kron IL, Kern JA. Preoperative computed tomography is associated with lower risk of perioperative stroke in reoperative cardiac surgery. Interact Cardiovasc Thorac Surg. 2011 Jun;12(6):919-23

10. Said SM, Dearani JA. Strategies for high-risk reoperations in congenital heart disease. Semin Thorac Cardiovasc Surg Pediatr Card Surg Annu. 2014;17(1):921

11. Kogon BE, Daniel W, Fay K, Book W. Is the liberal use of preoperative 3dimensional imaging and presternotomy femoral cutdown beneficial in reoperative adult congenital heart surgery?. J Thorac Cardiovasc Surg. 2014 Jun;147(6):1799-804

12. Kirmani BH, Brazier A, Sriskandarajah S, Azzam R, Keenan DJ. A meta-analysis of computerized tomography scan for reducing complications following repeat 
sternotomy for cardiac surgery. Interact Cardiovasc Thorac Surg. 2016 Apr;22(4):472-9

13.Akl BF, Pett SB Jr, Wernly JA. Use of a sagittal oscillating saw for repeat sternotomy: a safer and simpler technique. Ann Thorac Surg. 1984 Dec;38(6):646-7

14.Luciani N, Anselmi A, De Geest R, Martinelli L, Perisano M, Possati G. Extracorporeal circulation by peripheral cannulation before redo sternotomy: indications and results. J Thorac Cardiovasc Surg. 2008 Sep;136(3):572-7

15. Triedman JK, Newburger JW. Trends in Congenital Heart Disease: The Next Decade. Circulation. 2016 Jun;133(25):2716-33.

16. García A, Moreno K, Ronderos M, Sandoval N, Caicedo M, Dennis RJ. Differences by Altitude in the Frequency of Congenital Heart Defects in Colombia. Pediatric Cardiology. 2016 October; 37(7):1-9. 
Figure 1a.

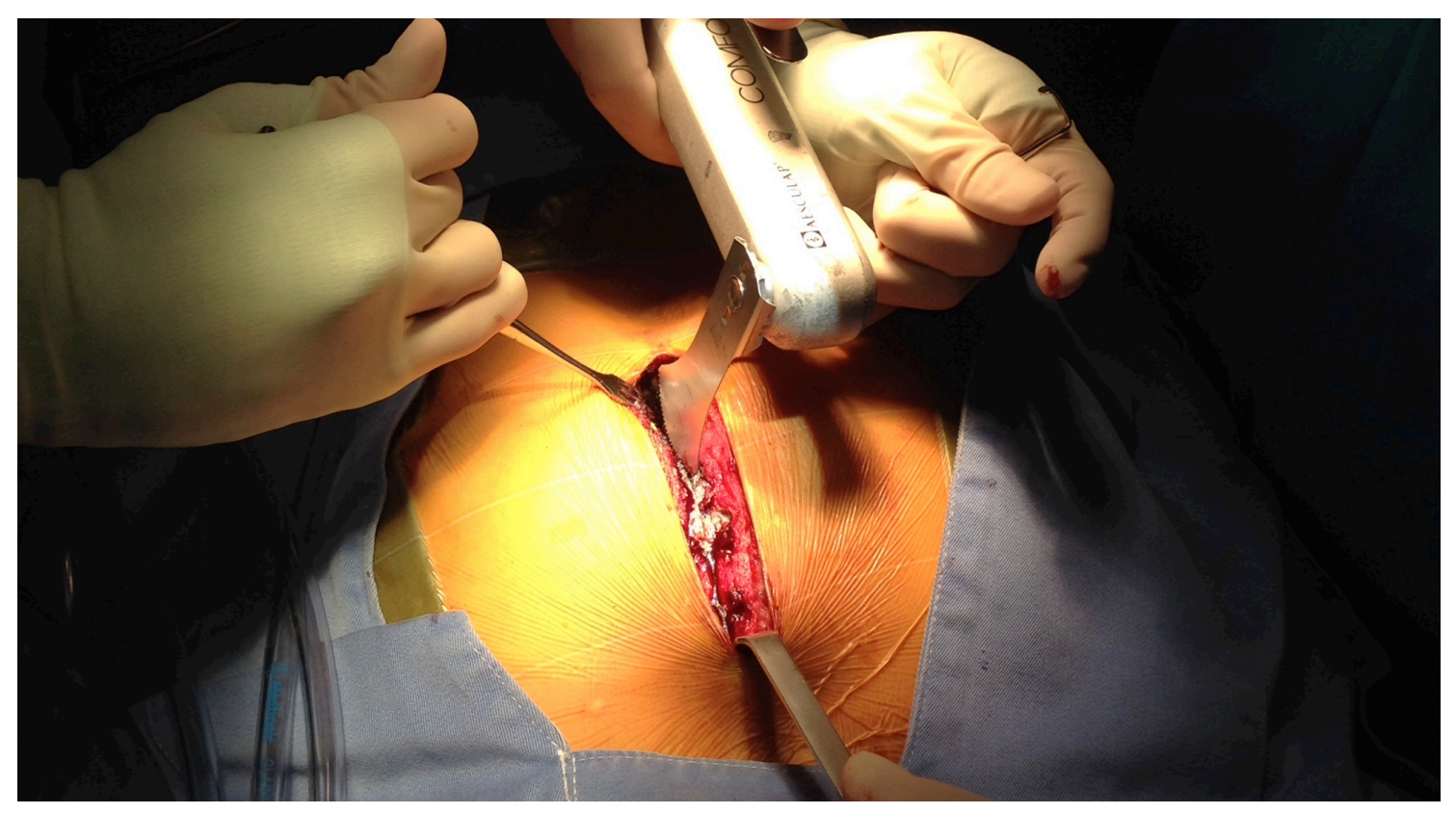


Figure 1b.

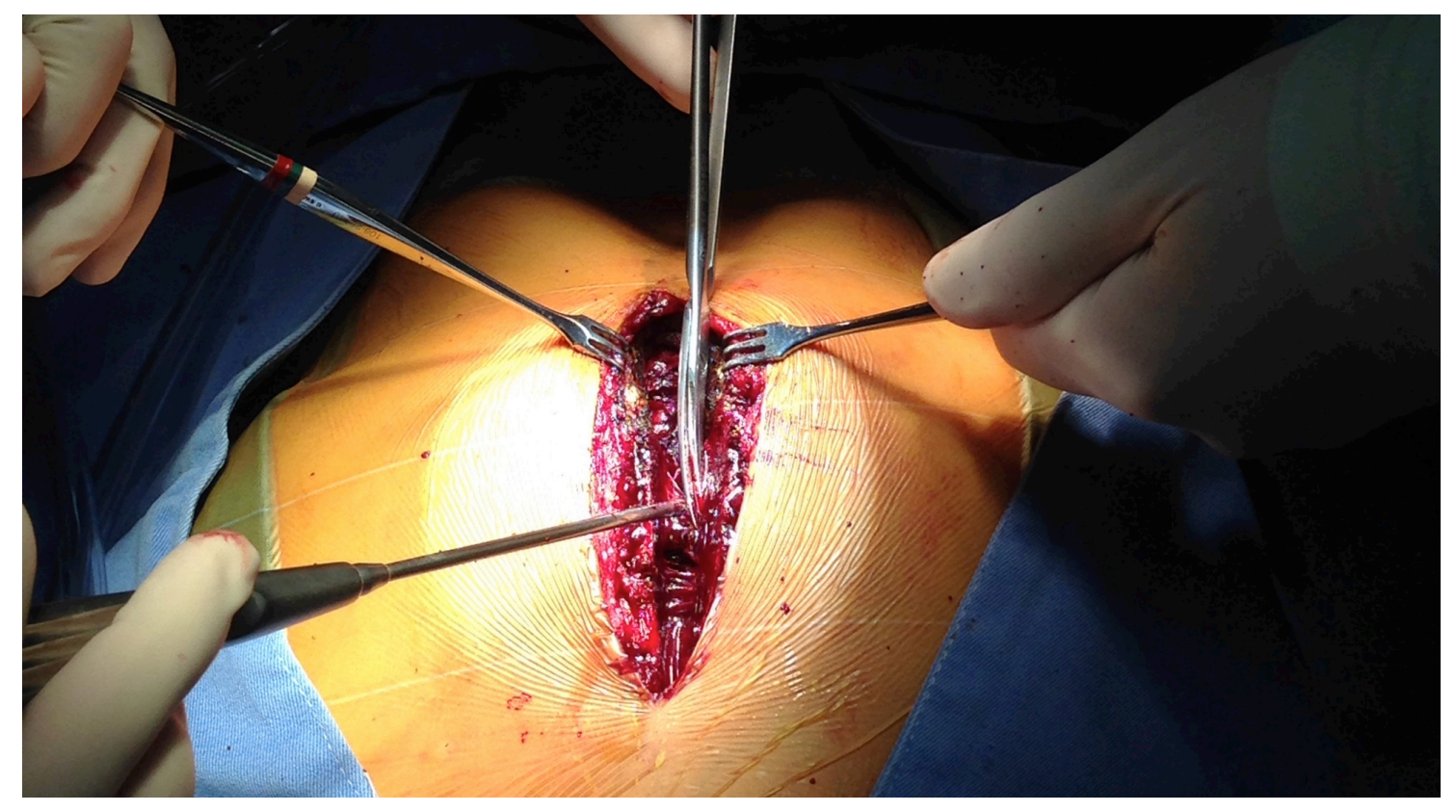


Figure 1c.

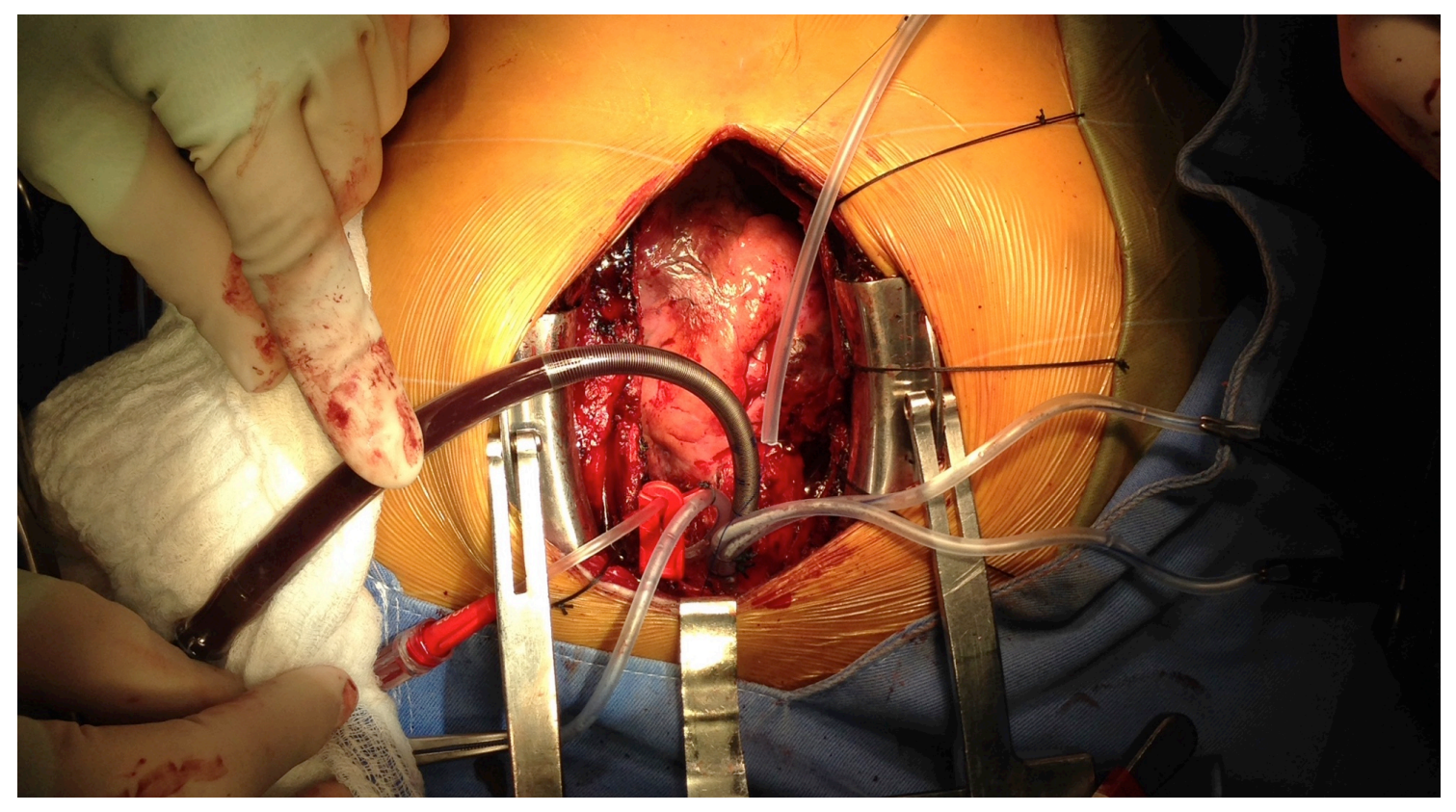


Figure 2. Reoperation tendency per year

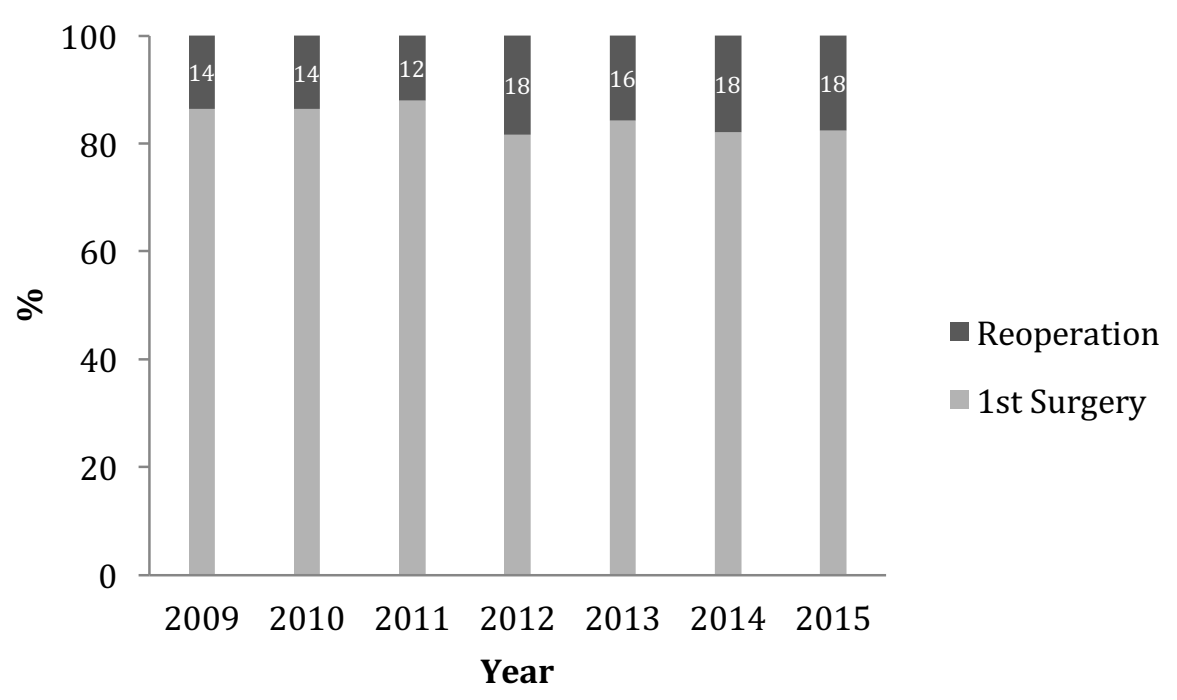


Table 1. Characteristics of patients.

\begin{tabular}{|c|c|c|c|c|}
\hline VARIABLE & $\begin{array}{l}\text { TOTAL } \\
\mathrm{N}=238\end{array}$ & $\begin{array}{l}\text { ALIVE } \\
N=227\end{array}$ & $\begin{array}{l}\text { DEAD } \\
N=11\end{array}$ & P VALUE \\
\hline Age, Years Median IQR & $6.4(2.9-12.2)$ & $6.6(2.9-12.3)$ & $2.9(0.4-9.6)$ & 0.11 \\
\hline Females, n (\%) & $107(44.9)$ & $101(44.9)$ & $6(54.5)$ & 0.51 \\
\hline Weight, Kg Median IQR & $18.8(11-33)$ & $19(11-33)$ & $11.9(7-25)$ & 0.13 \\
\hline Saturation, \% Median IQR & $92(85-94)$ & $92(85-94)$ & $90(79-96)$ & 0.79 \\
\hline Hematocrit, \%, Median IQR & $44(39-50)$ & $43(39-50)$ & $46(40-53)$ & 0.48 \\
\hline $\begin{array}{l}\text { Left ventricular ejection } \\
\text { fraction, \% Median IQR }\end{array}$ & $70(65-75)$ & $70(65-75)$ & $68(64-72)$ & 0.51 \\
\hline Down syndrome, $\mathbf{n}(\%)$ & $6(2.5)$ & $6(2.6)$ & 0 & 0.54 \\
\hline Number of surgeries $n(\%)$ & & & & 0.38 \\
\hline 1 & $168(70.6)$ & $159(70.0)$ & $9(81.8)$ & \\
\hline 2 & $54(22.7)$ & $53(23.3)$ & $1(9.1)$ & \\
\hline$\geq 3$ & $16(6.7)$ & $15(6.6)$ & $1(9.1)$ & \\
\hline CPB time, minutes Median IQR & $94(70-130)$ & $93(68-126)$ & $216(134-270)$ & $<0.000$ \\
\hline $\begin{array}{l}\text { Cross Clamp time, minutes } \\
\text { Median IQR }\end{array}$ & $66(45-97)$ & $63(45-86)$ & $128(102-170)$ & 0.0003 \\
\hline Open chest, $n$ (\%) & $21(8.8)$ & $15(6.6)$ & $6(54.5)$ & $<0.000$ \\
\hline STAT risk category & & & & 0.009 \\
\hline $1, n(\%)$ & $35(14.1)$ & $35(15.4)$ & 0 & \\
\hline $2, n(\%)$ & $107(44.9)$ & $104(45.8)$ & $3(27.3)$ & \\
\hline $3, \mathbf{n}(\%)$ & $25(10.5)$ & $23(10.1)$ & $2(18.2)$ & \\
\hline $4, n(\%)$ & $69(28.9)$ & $64(28.2)$ & $5(45.4)$ & \\
\hline $5, n(\%)$ & $2(0.8)$ & $1(0.4)$ & $1(9.1)$ & \\
\hline
\end{tabular}

$\mathrm{N}$ : number, \%: percentage, IQR: interquartile range, SD: standard deviation,KG: kilograms 
Table 2. Surgery Type.

\begin{tabular}{|l|l|l|}
\hline SURGERY TYPE & N & \multicolumn{2}{|l|}{$\%$} \\
\hline Fontan Fenestrated & 30 & 12,6 \\
\hline Pulmonary Valve Replacement & 24 & 10,1 \\
\hline Subaortic Membrane Resection & 18 & 7,6 \\
\hline Mitral Valve Replacement & 14 & 5,9 \\
\hline Glenn & 12 & 5,0 \\
\hline Aortic Valve Replacement & 12 & 5,0 \\
\hline TAPVC Correction & 11 & 4,6 \\
\hline Other & 117 & 49,2 \\
\hline
\end{tabular}


Table 3. Univariate risk factors for mortality.

\begin{tabular}{|l|c|c|c|}
\hline VARIABLE & OR & CI 95\% & P VALUE \\
\hline Age $<$ 4 years, yes & 3.8 & $1.1-13.3$ & 0.04 \\
\hline Cross clamp time > 100 minutes, yes & 15.5 & $1.9-123.2$ & 0.001 \\
\hline STAT risk category > 3 & 4.2 & $1.1-16.3$ & 0.04 \\
\hline
\end{tabular}

